\title{
Secure Transmission in MIMO-NOMA Networks
}

\author{
Yue Qi, Student Member, IEEE, and Mojtaba Vaezi, Senior Member, IEEE
}

\begin{abstract}
This letter focuses on the physical layer security over two-user multiple-input multiple-output (MIMO) non-orthogonal multiple access (NOMA) networks. A linear precoding technique is designed to ensure the confidentiality of the message of each user from its counterpart. This technique first splits the base station power between the two users and, based on that, decomposes the secure MIMO-NOMA channel into two MIMO wiretap channels, and designs the transmit covariance matrix for each channel separately. The proposed method substantially enlarges the secrecy rate compared to existing linear precoding methods and strikes a balance between performance and computation cost. Simulation results verify the effectiveness of the proposed method.
\end{abstract}

Index Terms-MIMO-NOMA, physical layer security, wiretap, precoding, GSVD.

\section{INTRODUCTION}

In view of its potential to increase connectivity, reduce latency, and improve spectral efficiency, non-orthogonal multiple access (NOMA) has attracted tremendous attention for fifth generation and beyond wireless networks [1]. In NOMA, the base station broadcasts the same signal to serve multiple users over the same resources in time/frequency/code/space. Due to the broadcast nature of transmission, NOMA users are susceptible to internal and external eavesdroppers. Therefore, new aspects of physical layer security need to be analyzed in NOMA networks.

To fulfill the security requirements of single-antenna NOMA networks, existing security techniques such as cooperated relaying and jamming have been proposed [2]-[5]. In multipleinput, multiple-output (MIMO) NOMA networks, other methods such as artificial noise (AN)-aided transmission and beamforming [6] [8] have been proposed to make communications less vulnerable to 'external' eavesdroppers. These solutions are mostly to secure data transmission from external eavesdroppers. However, since a superimposed signal is transmitted to a group of legitimate users, an important question is whether NOMA users can communicate their messages confidentially, or legitimate 'internal' users may compromise their security? Early works have proved that, in a two-user MIMO-NOMA network, both users can transmit their messages concurrently and confidentially via secret dirty-paper coding (S-DPC) [9] 1 The complexity of S-DPC is, however, not acceptable in practice. This motivates the development of low-complexity, fast solutions, such as designing linear precoding. In [11], a linear precoder based on generalized singular value decomposition (GSVD) is designed using orthogonal parallel channel transmission. However, this solution is far from the capacity region.

The authors are with the Department of Electrical and Computer Engineering, Villanova University, Villanova, PA 19085 USA (e-mail: yqi@villanova.edu; mvaezi@villanova.edu).

${ }^{1}$ There are other important information-theoretic models related to MIMONOMA security with external eavesdroppers [10].

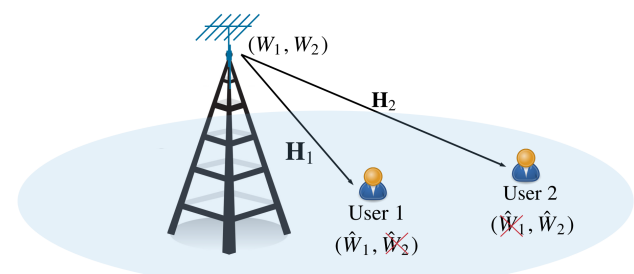

Fig. 1: Illustration of a two-user secure MIMO-NOMA network in which each user should be able to decode only its message.

In [12], the secrecy capacity of two-user MIMO-NOMA is transformed into a weighted secrecy sum-rate maximization problem. This method improves the achievable secrecy rates but still is time-consuming.

In this letter, we design precoding and power allocation matrices to achieve the secrecy capacity region of two-user Gaussian MIMO-NOMA networks under the total power constraints with reasonable time consumption. Due to the complexity of the problem, we decompose it into two wiretap channel subproblems. Then, we design the transmit covariance matrix for each problem and show that the performance loss is negligible.

The contributions of this letter are summarized as follows:

- By splitting the total power between the two users, we decompose the two-user secure MIMO-NOMA channel into two MIMO wiretap channels with respective power constraints.

- We propose new precoding and power allocation to solve the new problems, and introduce an efficient and costeffective algorithm for secure transmission in MIMONOMA networks within practical ranges of antennas. Our approach strikes a balance between performance and time consumption.

Notations: $\operatorname{tr}(\cdot)$ and $(\cdot)^{T}$ denote the trace and transpose of matrices. $\mathbb{E}(\cdot)$ denotes expectation. $\operatorname{diag}\left(\lambda_{1}, \ldots, \lambda_{n}\right)$ represents diagonal matrix with diagonal elements $\lambda_{1}, \ldots, \lambda_{n}$. $\mathbf{Q} \succcurlyeq \mathbf{0}$ means $\mathbf{Q}$ is a positive semidefinite matrix, and $\mathbf{I}$ is an identity matrix.

\section{SySTEM MODEL}

We consider a two-user MIMO-NOMA network, as shown in Fig. 1. The transmitter (Tx), user 1, and user 2 are equipped with $n_{t}, n_{1}$, and $n_{2}$ antennas, respectively. The Tx serves the users with two confidential messages $W_{1}$ and $W_{2}$ (such as accessing bank accounts and performing online transactions), i.e., user $i$ should not be able to decode $W_{i}$ when $i \neq j, i, j \in$ $\{1,2\}$. In this setting, user 1 can be seen as an eavesdropper to user 2 and vice versa. Due to NOMA transmission, the input vectors $x_{1} \in \mathbb{R}^{n_{t} \times 1}$ and $x_{2} \in \mathbb{R}^{n_{t} \times 1}$ intended for user 1 and 
user 2 share the same time and frequency slot. The received signals at user 1 and user 2 , respectively, are given by

$$
\begin{aligned}
& \mathbf{y}_{1}=\mathbf{H}_{1}\left(\mathbf{x}_{1}+\mathbf{x}_{2}\right)+\mathbf{w}_{1}, \\
& \mathbf{y}_{2}=\mathbf{H}_{2}\left(\mathbf{x}_{1}+\mathbf{x}_{2}\right)+\mathbf{w}_{2},
\end{aligned}
$$

in which $\mathbf{H}_{1} \in \mathbb{R}^{n_{1} \times n_{t}}$ and $\mathbf{H}_{2} \in \mathbb{R}^{n_{2} \times n_{t}}$ are the channel matrices for user 1 and user 2 , and $\mathbf{w}_{1} \in \mathbb{R}^{n_{1} \times 1}$ and $\mathbf{w}_{2} \in$ $\mathbb{R}^{n_{2} \times 1}$ are independent identically distributed (i.i.d) Gaussian noise vectors whose elements are zero mean and unit variance.

This setting is also known as MIMO broadcast channel (BC) with two confidential messages, and its secrecy capacity region under the average total power constraint can be expressed as [9], [13]

$$
\begin{array}{r}
R_{1} \leq \frac{1}{2} \log \left|\mathbf{I}+\mathbf{H}_{1} \mathbf{Q}_{1} \mathbf{H}_{1}^{T}\right|-\frac{1}{2} \log \left|\mathbf{I}+\mathbf{H}_{2} \mathbf{Q}_{1} \mathbf{H}_{2}^{T}\right|, \quad \text { (2a) } \\
R_{2} \leq \frac{1}{2} \log \left|\mathbf{I}+\frac{\mathbf{H}_{2} \mathbf{Q}_{2} \mathbf{H}_{2}^{T}}{\mathbf{I}+\mathbf{H}_{2} \mathbf{Q}_{1} \mathbf{H}_{2}^{T}}\right|-\frac{1}{2} \log \left|\mathbf{I}+\frac{\mathbf{H}_{1} \mathbf{Q}_{2} \mathbf{H}_{1}^{T}}{\mathbf{I}+\mathbf{H}_{1} \mathbf{Q}_{1} \mathbf{H}_{1}^{T}}\right| \\
\text { s.t. } \operatorname{tr}\left(\mathbf{Q}_{1}+\mathbf{Q}_{2}\right) \leq P, \mathbf{Q}_{1} \succcurlyeq \mathbf{0}, \mathbf{Q}_{2} \succcurlyeq \mathbf{0}
\end{array}
$$

in which $\mathbf{Q}_{1}=\mathbb{E}\left(\mathbf{x}_{1} \mathbf{x}_{1}^{T}\right)$ and $\mathbf{Q}_{2}=\mathbb{E}\left(\mathbf{x}_{2} \mathbf{x}_{2}^{T}\right)$ are the input covariance matrices corresponding to $\mathrm{x}_{1}$ and $\mathrm{x}_{2}$, respectively.

The capacity region in (2) is obtained via S-DPC. However, S-DPC is prohibitively complex for practical uses. Typically, an exhaustive search over all possible $\mathbf{Q}_{1}$ and $\mathbf{Q}_{2}$ satisfying the constraints in (2c) [9] is used to get the capacity region. In [11], a GSVD-base precoder is proposed for this channel. Although its complexity is low, the rate region of GSVD-based precoding is far from the capacity region.

In this letter, we show that the above secure MIMONOMA channel can be seen as two interwoven MIMO wiretap channels. In one wiretap channel, user 1 is viewed as a legitimate user, while user 2 is an eavesdropper. The secrecy rate of this channel is obtained from (2a). In the second wiretap channel, the role of user 1 and user 2 is swapped, and the secrecy rate of this channel is obtained from (2b). Due to the symmetry of the channel, the rate region in (2) can be equivalently obtained by swapping the subscripts 1 and 2 in (2a) and (2b) [13, Corollary 1]. Next, we design novel precoding and power allocation schemes that achieve capacity region with reasonable complexity.

\section{Decomposing SECURE MiMO-NOMA INTO Two MIMO WIRETAP CHANNELS}

In order to introduce new simpler solutions, in this section, we decompose the aforementioned secure MIMO-NOMA channel into two MIMO wiretap channels. This is done in three steps. First, similar to the BC channel, we split the power between the two users. Then, we decouple the secure MIMONOMA channel into two MIMO wiretap channels to solve them separately, as described below.

Step 1: Introducing power splitting factor $\alpha \in[0,1]$, we dedicate a fraction $\alpha$ of the total power to user $1\left(P_{1}=\alpha P\right)$, and fraction $\bar{\alpha}, \bar{\alpha}=1-\alpha$, to user $2\left(P_{2}=\bar{\alpha} P\right)$.
Step 2: We design secure precoding for user 1 while treating user 2 as an eavesdropper. Because (2a) is only controlled by the covariance matrix $\mathbf{Q}_{1}$, the problem can be seen as a wiretap channel under a transmit power $P_{1}$, which is

$$
\begin{gathered}
R_{1}(\alpha)=\max _{\mathbf{Q}_{1} \succeq \mathbf{0}} \frac{1}{2} \log \frac{\left|\mathbf{I}+\mathbf{H}_{1} \mathbf{Q}_{1} \mathbf{H}_{1}^{T}\right|}{\left|\mathbf{I}+\mathbf{H}_{2} \mathbf{Q}_{1} \mathbf{H}_{2}^{T}\right|}, \\
\text { s.t. } \operatorname{tr}\left(\mathbf{Q}_{1}\right) \leq P_{1}=\alpha P .
\end{gathered}
$$

This problem is now the well-known MIMO wiretap channel [14], and standard MIMO wiretap solutions can be applied.

Step 3: We design secure precoding for user 2 to maximize the rate of user 2 by allocating the remaining power, and using $\mathrm{Q}_{1}^{*}$ obtained in Step 2 to (2b). Thus, 2b is represented as

$$
\begin{aligned}
& R_{2}(\alpha)=\max _{\mathbf{Q}_{2} \succeq \mathbf{0}}\left\{\frac{1}{2} \log \left|\mathbf{I}+\frac{\mathbf{H}_{2} \mathbf{Q}_{2} \mathbf{H}_{2}^{T}}{\mathbf{I}+\mathbf{H}_{2} \mathbf{Q}_{1}^{*} \mathbf{H}_{2}^{T}}\right|\right. \\
& \left.\quad-\frac{1}{2} \log \left|\mathbf{I}+\frac{\mathbf{H}_{1} \mathbf{Q}_{2} \mathbf{H}_{1}^{T}}{\mathbf{I}+\mathbf{H}_{1} \mathbf{Q}_{1}^{*} \mathbf{H}_{1}^{T}}\right|\right\}, \\
& \text { s.t. } \operatorname{tr}\left(\mathbf{Q}_{2}\right) \leq P_{2}=(1-\alpha) P .
\end{aligned}
$$

Since $\mathbf{Q}_{1}^{*}$ is given after solving (3), in the following we show that the above problem can be seen as another wiretap channel where users 2 and 1 are the legitimate user and eavesdropper, respectively.

Theorem 1. The above channel can be converted to a standard MIMO wiretap channel with

$$
\begin{aligned}
& \mathbf{H}_{1}^{\prime} \triangleq \boldsymbol{\Lambda}_{a}^{-\frac{1}{2}} \mathbf{V}_{a}^{T} \mathbf{H}_{1}, \\
& \mathbf{H}_{2}^{\prime} \triangleq \boldsymbol{\Lambda}_{b}^{-\frac{1}{2}} \mathbf{V}_{b}^{T} \mathbf{H}_{2},
\end{aligned}
$$

in which $\boldsymbol{\Lambda}_{a}$ and $\mathbf{V}_{a}$ are the eigenvalues and eigenvectors of $\mathbf{I}+\mathbf{H}_{1} \mathbf{Q}_{1}^{*} \mathbf{H}_{1}^{T}$, and $\boldsymbol{\Lambda}_{b}$ and $\mathbf{V}_{b}$ are the eigenvalues and eigenvectors of $\mathbf{I}+\mathbf{H}_{2} \mathbf{Q}_{1}^{*} \mathbf{H}_{2}^{T}$.

Proof. Let us define

$$
\begin{aligned}
& \boldsymbol{\Sigma}_{1} \triangleq \mathbf{I}+\mathbf{H}_{1} \mathbf{Q}_{1}^{*} \mathbf{H}_{1}^{T}=\mathbf{V}_{a} \boldsymbol{\Lambda}_{a} \mathbf{V}_{a}^{T} \\
& \boldsymbol{\Sigma}_{2} \triangleq \mathbf{I}+\mathbf{H}_{2} \mathbf{Q}_{1}^{*} \mathbf{H}_{2}^{T}=\mathbf{V}_{b} \boldsymbol{\Lambda}_{b} \mathbf{V}_{b}^{T}
\end{aligned}
$$

Then, the rate for user 2 can be written as

$$
\begin{aligned}
R_{2}(\alpha) & =\max _{\mathbf{Q}_{2} \succeq \mathbf{0}} \frac{1}{2} \log \frac{\left|\mathbf{I}+\mathbf{H}_{2} \mathbf{Q}_{2} \mathbf{H}_{2}^{T} \boldsymbol{\Sigma}_{2}^{-1}\right|}{\left|\mathbf{I}+\mathbf{H}_{1} \mathbf{Q}_{2} \mathbf{H}_{1}^{T} \mathbf{\Sigma}_{1}^{-1}\right|} \\
& =\max _{\mathbf{Q}_{2} \succeq \mathbf{0}} \frac{1}{2} \log \frac{\left|\mathbf{I}+\mathbf{H}_{2} \mathbf{Q}_{2} \mathbf{H}_{2}^{T} \mathbf{V}_{b} \boldsymbol{\Lambda}_{b}^{-1} \mathbf{V}_{b}^{T}\right|}{\left|\mathbf{I}+\mathbf{H}_{1} \mathbf{Q}_{2} \mathbf{H}_{1}^{T} \mathbf{V}_{a} \boldsymbol{\Lambda}_{a}^{-1} \mathbf{V}_{a}^{T}\right|} \\
& \stackrel{(a)}{=} \max _{\mathbf{Q}_{2} \succeq \mathbf{0}} \frac{1}{2} \log \frac{\left|\mathbf{I}+\boldsymbol{\Lambda}_{b}^{-\frac{1}{2}} \mathbf{V}_{b}^{T} \mathbf{H}_{2} \mathbf{Q}_{2} \mathbf{H}_{2}^{T} \mathbf{V}_{b} \boldsymbol{\Lambda}_{b}^{-\frac{1}{2}}\right|}{\left|\mathbf{I}+\mathbf{\Lambda}_{a}^{-\frac{1}{2}} \mathbf{V}_{a}^{T} \mathbf{H}_{1} \mathbf{Q}_{2} \mathbf{H}_{1}^{T} \mathbf{V}_{a} \boldsymbol{\Lambda}_{a}^{-\frac{1}{2}}\right|} \\
& =\max _{\mathbf{Q}_{2} \succeq \mathbf{0}} \frac{1}{2} \log \frac{\left|\mathbf{I}+\mathbf{H}_{2}^{\prime} \mathbf{Q}_{2} \mathbf{H}_{2}^{\prime T}\right|}{\left|\mathbf{I}+\mathbf{H}_{1}^{\prime} \mathbf{Q}_{2} \mathbf{H}_{1}^{\prime T}\right|},
\end{aligned}
$$

in which $(a)$ holds because $\operatorname{det}(\mathbf{I}+\mathbf{A B})=\operatorname{det}(\mathbf{I}+\mathbf{B A})$ and $\boldsymbol{\Lambda}_{a}$ and $\boldsymbol{\Lambda}_{b}$ are diagonal matrices.

In view of (7), it is seen that like (3a), 4a is the rate for a MIMO wiretap channel with channels $\mathbf{H}_{2}^{\prime}$ for the legitimate user and $\mathbf{H}_{1}^{\prime}$ for the eavesdropper. 


\section{Secure Precoding and Power Allocation}

In this section, we propose new linear precoding and power allocation strategies to secure the MIMO-NOMA channel. In light of our decomposition in the previous section, we have two MIMO wiretap channels and thus standard MIMO wiretap solutions can be applied to design covariance matrices $\mathbf{Q}_{1}$ and $\mathbf{Q}_{2}$. One fast approach is rotation based linear precoding [14]. In this method, the covariance matrix $\mathbf{Q}_{1}$ is eigendecomposed into one rotation matrix $\mathbf{V}_{1}$ and one power allocation matrix $\Lambda_{1}$ [14], [15] as

$$
\mathbf{Q}_{1}=\mathbf{V}_{1} \mathbf{\Lambda}_{1} \mathbf{V}_{1}^{T}
$$

Consequently, the secrecy capacity of user 1 is

$$
\begin{aligned}
R_{1}(\alpha) & =\max _{\mathbf{Q}_{1} \succeq \mathbf{0}} \frac{1}{2} \log \frac{\left|\mathbf{I}+\mathbf{H}_{1} \mathbf{V}_{1} \mathbf{\Lambda}_{1} \mathbf{V}_{1}^{T} \mathbf{H}_{1}^{T}\right|}{\left|\mathbf{I}+\mathbf{H}_{2} \mathbf{V}_{1} \boldsymbol{\Lambda}_{1} \mathbf{V}_{1}^{T} \mathbf{H}_{2}^{T}\right|}, \\
\text { s.t. } & \sum_{k=1}^{n_{t}} \lambda_{1 k} \leq P_{1}=\alpha P,
\end{aligned}
$$

in which $\lambda_{1 k}, k=\left\{1, \ldots, n_{t}\right\}$, is a diagonal element of matrix $\boldsymbol{\Lambda}_{1}=\operatorname{diag}\left(\lambda_{11}, \ldots, \lambda_{1 n_{t}}\right)$. The rotation matrix $\mathbf{V}_{1}$ can be obtained by

$$
\mathbf{V}_{1}=\prod_{i=1}^{n_{t}-1} \prod_{j=i+1}^{n_{t}} \mathbf{V}_{i j}
$$

in which the basic rotation matrix $\mathbf{V}_{i j}$ is a Givens matrix [16] which is an identity matrix except that its elements in the $i$ th row and $j$ th column, i.e., $v_{i i}, v_{i j}, v_{j i}$, and $v_{j j}$ are replaced by

$$
\left[\begin{array}{ll}
v_{i i} & v_{i j} \\
v_{j i} & v_{j j}
\end{array}\right]=\left[\begin{array}{cc}
\cos \theta_{1 i j} & -\sin \theta_{1 i j} \\
\sin \theta_{1 i j} & \cos \theta_{1 i j}
\end{array}\right],
$$

where $\theta_{1 i j}$ is rotation angle corresponding to the rotation matrix $\mathbf{V}_{i j}$. Then, we will optimize the new parameterized nonconvex problem numerically to obtain the solution $\mathrm{Q}_{1}^{*}$ with respect to rotation angles and power allocation parameters.

Similarly, covariance matrix $\mathbf{Q}_{2}$ can be written by rotation method as $\mathbf{Q}_{2}=\mathbf{V}_{2} \boldsymbol{\Lambda}_{2} \mathbf{V}_{2}^{T}$, where the rotation matrix $\mathbf{V}_{2}$ is defined similar to $\mathbf{V}_{1}$ in (10) with its rotation angles are $\theta_{2 i j}$. Therefore, the optimization problem for $R_{2}(\alpha)$ becomes

$$
\begin{aligned}
R_{2}(\alpha) & =\max _{\mathbf{Q}_{2} \succeq \mathbf{0}} \frac{1}{2} \log \frac{\left|\mathbf{I}+\mathbf{H}_{1}^{\prime} \mathbf{V}_{2} \boldsymbol{\Lambda}_{2} \mathbf{V}_{2}^{T} \mathbf{H}_{1}^{\prime T}\right|}{\left|\mathbf{I}+\mathbf{H}_{2}^{\prime} \mathbf{V}_{2} \boldsymbol{\Lambda}_{2} \mathbf{V}_{2}^{T} \mathbf{H}_{2}^{\prime T}\right|}, \\
\text { s.t. } \quad & \sum_{k=1}^{n_{t}} \lambda_{2 k} \leq P_{2}=(1-\alpha) P,
\end{aligned}
$$

in which $\lambda_{2 k}$ is the $k$ th diagonal element of $\boldsymbol{\Lambda}_{2}$. This problem is again similar to (9).

To solve the new parameterized problems in (9) and (12) to find new parameters $\lambda_{1 k}, \theta_{1 i j}$ and $\lambda_{2 k}, \theta_{2 i j}$ (instead of directly finding $\mathbf{Q}_{1}$ and $\mathbf{Q}_{2}$ in (2)), various numerical approaches such as Matlab fmincon can be used. In this paper, for a fixed $\alpha \in[0,1]$, we use Broyden-Fletcher-Goldfarb-Shanno (BFGS) method together with the interior-point method (IPM) [17]. IPM transfers the constraints into an unconstrained problem, and BFGS is a quasi-Newton iterative method for nonlinear optimization. The algorithm is elaborated in Algorithm 1.

In the power splitting method, we introduced in Section III and new optimization problems in (9) and (12), for each $\alpha$,

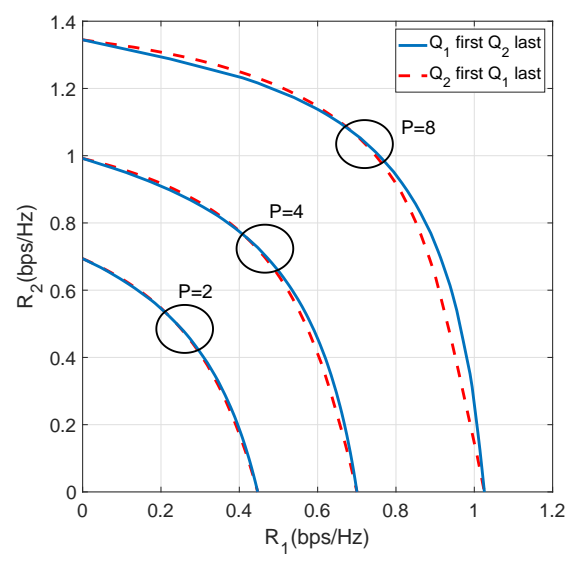

Fig. 2: The effect of the order of precoding on the secure rate region for different values of transmit power $(P)$.

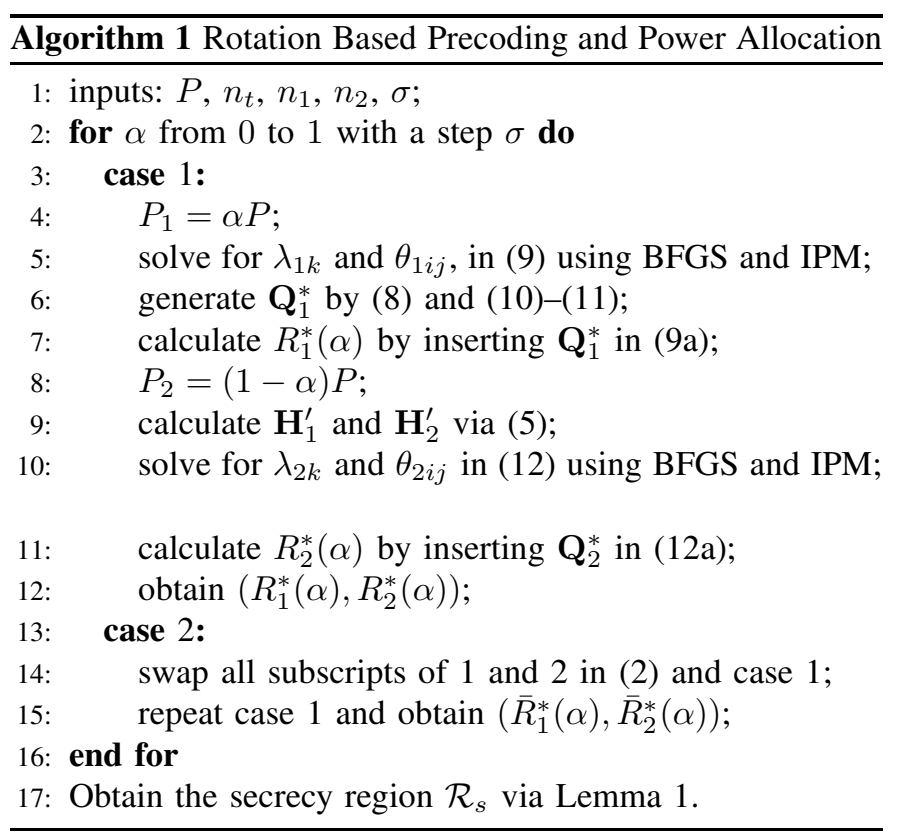

we solve for $\mathbf{Q}_{1}^{*}$ and $\mathbf{Q}_{2}^{*}$ (and thus $R_{1}^{*}(\alpha)$ and $R_{2}^{*}(\alpha)$ ) step by step. This simplifies the problem but may result in sub-optimal rate region. Moreover, the order of optimization (first $R_{1}^{*}$ then $R_{2}^{*}$ ) will affect the solution.

Alternatively, we can first solve for $\mathbf{Q}_{2}^{*}$ followed by $\mathbf{Q}_{1}^{*}$ (i.e., first $R_{2}^{*}$ then $\left.R_{1}^{*}\right)$. We represent this solution $\left(\bar{R}_{1}^{*}(\alpha), \bar{R}_{2}^{*}(\alpha)\right)$ in Algorithm 1 In general, changing the order of optimization will result in a different rate region. To show how the order of precoding can change the achievable rate region, we demonstrate an example in Fig. 2 with different powers $P=2,4,8$, where the channels are

$$
\begin{aligned}
& \mathbf{H}_{1}=\left[\begin{array}{lll}
0.125 & 0.821 & 0.087 \\
0.383 & 0.261 & 0.037
\end{array}\right] \\
& \mathbf{H}_{2}=\left[\begin{array}{lll}
0.384 & 0.703 & 0.849
\end{array}\right]
\end{aligned}
$$

Thus, the convex hull of the two solutions with different orders 


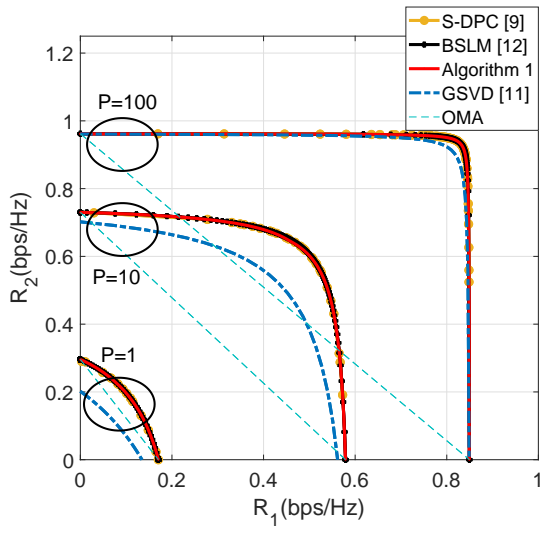

Fig. 3: Secrecy rate regions for $n_{t}=n_{1}=n_{2}=2$ with different powers.

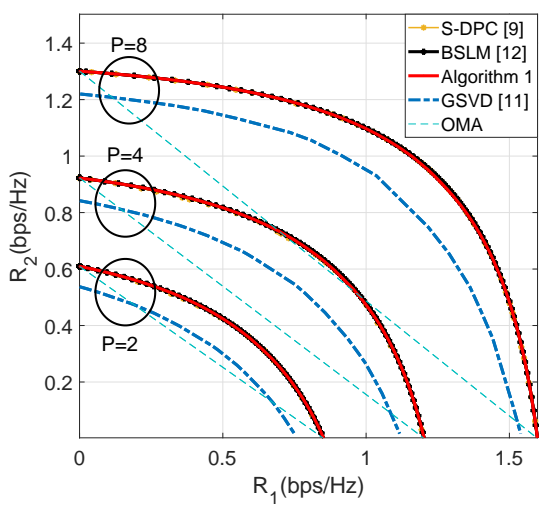

Fig. 4: Secrecy rate regions for $n_{t}=3, n_{1}=n_{2}=2$ with different powers.

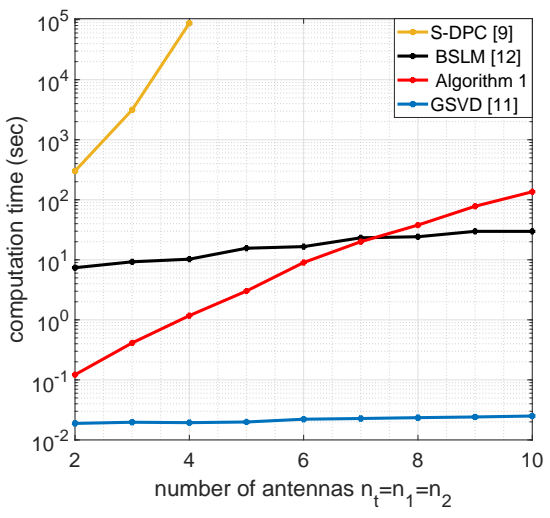

Fig. 5: Computational time versus number of antennas for $P=30$. may enlarge the achievable rate region.

Lemma 1. The achievable secrecy region $\mathcal{R}_{s}$ for the secure MIMO-NOMA channel under total power constraint is the convex hull of all rate points

$$
\mathcal{R}_{s}=\bigcup_{0 \leq \alpha \leq 1}\left\{\left(R_{1}^{*}(\alpha), R_{2}^{*}(\alpha)\right) \cup\left(\bar{R}_{1}^{*}(\alpha), \bar{R}_{2}^{*}(\alpha)\right)\right\},
$$

where $\left(R_{1}^{*}(\alpha), R_{2}^{*}(\alpha)\right)$ is obtained by precoding for user 1 then user 2 , whereas $\left(\bar{R}_{1}^{*}(\alpha), \bar{R}_{2}^{*}(\alpha)\right)$ is obtained by precoding in the reverse order (first user 2, then user 1 ).

Remark 1 (Complexity Analysis): The BFGS algorithm yields the complexity of $\mathcal{O}\left(n^{2}\right)$ [17], where $n$ is the size of input variables which in our case is the number of rotation parameters, i.e., $n=\frac{\left(n_{t}+1\right) n_{t}}{2}$. On the other hand, the computation of matrix multiplications and matrix inverse yield the complexity of $\mathcal{O}\left(L^{3}\right)$ where $L=\max \left(n_{t}, n_{1}, n_{2}\right)$. Thus, the overall complexity of Algorithm 1 is $\mathcal{O}\left(\frac{n_{t}^{4}+L^{3}}{\sigma}\right)$ and $\sigma$ is the search step of the power fraction $\alpha$. The weighted sum-rate has the complexity of $\mathcal{O}\left(\frac{L^{3}}{\epsilon \sigma} \log (1 / \epsilon)\right)$ with a search over the weight [12], and $\epsilon$ is the convergence tolerance of algorithm. The GSVD-based precoding [11] has $\mathcal{O}\left(\frac{L^{3}}{\sigma}+\frac{L}{\sigma} \log (1 / \epsilon)\right)$, in which the search step $\sigma$ over power comes from [11, Corollary 1]. The computational complexity of the exhaustive search for S-DPC is exponential in $L[9]$.

\section{NUMERICAL RESULTS}

In this section, we compare the rate region $\left(R_{1}, R_{2}\right)$ of the proposed method with the secrecy capacity region obtained by S-DPC [9], weighted secrecy sum-rate maximization using the block successive lower-bound maximization (BSLM) [12], and GSVD-based precoding [11]. The capacity region is obtained by an exhaustive search over all input covariance matrices whereas BSLM maximization is achieved by an iterative algorithm, which successively optimizes a sequence of approximated functions using binary search [12] to reach higher rates. GSVD-based precoding is an orthogonal channel assignment, and orthogonal multiple access (OMA) is achieved by the time-sharing between the two extreme points (two wiretap channels) which realizes the same task in two orthogonal time slots controlled by the time-sharing fraction.

The channel matrices $\mathbf{H}_{1}$ and $\mathbf{H}_{2}$ are generated randomly

$$
\mathbf{H}_{1}=\left[\begin{array}{ll}
0.783 & 0.590 \\
0.734 & 0.092
\end{array}\right], \quad \mathbf{H}_{2}=\left[\begin{array}{ll}
0.244 & 0.617 \\
0.947 & 0.807
\end{array}\right],
$$

for $P=1,10,100$. The search step $\epsilon$ for Algorithm 1 and GSVD-based precoding [11] is set as 0.05 identically. As Fig. 3 illustrates, the proposed algorithm achieves larger rate regions compared to GSVD-based precoding [11], and almost identical to the capacity and BSLM maximization [12]. In the MISO case of $\mathbf{H}_{1}=\left[\begin{array}{ll}1.5 & 0\end{array}\right]$ and $\mathbf{H}_{1}=$ [1.801 0.871], and $P=10,100$, and 1000 , we found that our proposed algorithm can reach the same secrecy region and obtained exactly the same figure in [12, Fig. 2].

Fig. 4 shows secrecy rate regions for $n_{t}=3$ with $P=$ $2,4,8$, and $\sigma=0.05$, where the channels are

$$
\begin{aligned}
\mathbf{H}_{1} & =\left[\begin{array}{lll}
0.813 & 0.232 & 0.085 \\
0.842 & 0.130 & 0.203
\end{array}\right], \\
\mathbf{H}_{2} & =\left[\begin{array}{lll}
0.315 & 0.769 & 0.294 \\
0.025 & 0.271 & 0.281
\end{array}\right] .
\end{aligned}
$$

Again, the proposed method largely outperforms GSVDbased precoding, and achieve the same rate region compared with the BSLM method.

Although both BSLM and the proposed method are very close to the secrecy capacity, the computation costs of the two methods are remarkably different, particularly for practical numbers of antennas. Table. I, Table. [I] and Fig. [5 show the execution time for all three precoding methods over 100 random channel realizations. BSLM takes much higher time to reach the same rate region as our proposed method in Algorithm 11 as BSLM is achieved by an iterative algorithm. Our proposed method can save time ten to a hundred times especially for portable devices equipped with a few antennas and can strike a balance between performance and computation complexity.

For massive MIMO-NOMA, the number of optimization parameters in Algorithm 1 become considerable as $n_{t}$ becomes very large. The solution proposed in this letter is not meant for such scenarios. One can, however, reduce the complexity for 
TABLE I: Execution time (ms) for $n_{t}=2$ and $P=30$.

\begin{tabular}{|c|c|c|c|c|c|c|}
\hline \multirow{2}{*}{\multicolumn{2}{|c|}{ BSLM [12] }} & \multicolumn{5}{|c|}{$n_{2}$} \\
\hline & & 1 & 2 & 3 & 4 & 5 \\
\hline \multirow{5}{*}{$n_{1}$} & 1 & 2063.0 & 3428.2 & 3774.0 & 4904 & 4516.6 \\
\hline & 2 & 3735.1 & 6524.8 & 7411.6 & 8630.8 & 9400.4 \\
\hline & 3 & 4814.3 & 7158.5 & 9675.9 & 10310.4 & 10411.4 \\
\hline & 4 & 5426.1 & 9380.0 & 11285.4 & 13446.8 & 13086.3 \\
\hline & 5 & 6776.4 & 10931.0 & 13572.1 & 15300.5 & 16959.1 \\
\hline \multirow{2}{*}{\multicolumn{2}{|c|}{ Algorithm 1}} & \multicolumn{5}{|c|}{$n_{2}$} \\
\hline & & 1 & 2 & 3 & 4 & 5 \\
\hline \multirow{5}{*}{$n_{1}$} & 1 & 113.7 & 112.2 & 101.8 & 102.3 & 95.3 \\
\hline & 2 & 108.2 & 105.5 & 99.9 & 98.1 & 93.5 \\
\hline & 3 & 95.0 & 100.2 & 101.9 & 99.0 & 96.0 \\
\hline & 4 & 94.6 & 98.9 & 106.6 & 99.1 & 101.2 \\
\hline & 5 & 94.2 & 92.9 & 98.4 & 96.8 & 95.6 \\
\hline \multirow{2}{*}{\multicolumn{2}{|c|}{ GSVD [11] }} & \multicolumn{5}{|c|}{$n_{2}$} \\
\hline & & 1 & 2 & 3 & 4 & 5 \\
\hline \multirow{5}{*}{$n_{1}$} & 1 & 16.9 & 17.0 & 16.5 & 17.0 & 16.6 \\
\hline & 2 & 16.8 & 17.0 & 17.3 & 16.9 & 17.1 \\
\hline & 3 & 16.6 & 17.3 & 17.2 & 17.6 & 17.8 \\
\hline & 4 & 16.8 & 17.2 & 18.6 & 17.8 & 19.1 \\
\hline & 5 & 18.1 & 17.3 & 17.6 & 18.0 & 17.7 \\
\hline
\end{tabular}

TABLE II: Execution time (ms) for $n_{t}=3$ and $P=30$.

\begin{tabular}{|c|c|c|c|c|c|c|}
\hline \multirow{2}{*}{\multicolumn{2}{|c|}{ BSLM [12] }} & \multicolumn{5}{|c|}{$n_{2}$} \\
\hline & & 1 & 2 & 3 & 4 & 5 \\
\hline \multirow{5}{*}{$n_{1}$} & 1 & 2364.8 & 3029.8 & 4218.9 & 4893.1 & 4776.5 \\
\hline & 2 & 3386.5 & 4612.6 & 6138.8 & 7504.0 & 9829.3 \\
\hline & 3 & 5304.3 & 8190.1 & 10169.9 & 11428.9 & 12051.0 \\
\hline & 4 & 5432.4 & 7982.4 & 10363.0 & 13440.3 & 13744.5 \\
\hline & 5 & 6708.0 & 8450.5 & 11337.1 & 13851.8 & 15552.5 \\
\hline \multirow{2}{*}{\multicolumn{2}{|c|}{ Algorithm 1}} & \multicolumn{5}{|c|}{$n_{2}$} \\
\hline & & 1 & 2 & 3 & 4 & 5 \\
\hline \multirow{5}{*}{$n_{1}$} & 1 & 215.5 & 503.2 & 476.0 & 448.6 & 409.1 \\
\hline & 2 & 493.2 & 399.9 & 375.7 & 379.9 & 371.8 \\
\hline & 3 & 478.8 & 381.7 & 354.0 & 392.6 & 396.0 \\
\hline & 4 & 428.5 & 364.0 & 379.8 & 407.9 & 388.1 \\
\hline & 5 & 460.7 & 391.3 & 377.8 & 370.6 & 371.9 \\
\hline \multirow{2}{*}{\multicolumn{2}{|c|}{ GSVD [11 }} & \multicolumn{5}{|c|}{$n_{2}$} \\
\hline & & 1 & 2 & 3 & 4 & 5 \\
\hline \multirow{5}{*}{$n_{1}$} & 1 & 17.1 & 19.6 & 17.8 & 18.1 & 18.6 \\
\hline & 2 & 19.5 & 18.9 & 18.2 & 19.4 & 19.6 \\
\hline & 3 & 18.2 & 18.0 & 18.4 & 22.0 & 21.6 \\
\hline & 4 & 18.7 & 19.4 & 21.1 & 23.4 & 22.6 \\
\hline & 5 & 23.2 & 22.2 & 21.2 & 22.5 & 23.3 \\
\hline
\end{tabular}

large values of $n_{t}$ by applying other wiretap solutions such as alternating optimization [18]. On the other hand, other massive MIMO solutions, such as [8], [19], [20] may be more effective.

\section{CONCLUSIONS}

A novel linear precoding has been proposed for secure transmission over MIMO-NOMA networks to prevent users from eavesdropping each other. The proposed approach decomposes the two-user MIMO-NOMA channel into two MIMO wiretap channels via splitting the base station power between the two users and modifying the channel corresponding to one of the users to make it a wiretap channel in effect. This approach achieves a significantly higher secure rate region compared to existing linear precoding, and has an acceptable computational complexity.

\section{REFERENCES}

[1] M. Vaezi, G. A. A. Baduge, Y. Liu, A. Arafa, F. Fang, and Z. Ding, "Interplay between NOMA and other emerging technologies: A survey," IEEE Transactions on Cognitive Communications and Networking, vol. 5, no. 4, pp. 900-919, 2019.

[2] Y. Liu, Z. Qin, M. Elkashlan, Y. Gao, and L. Hanzo, "Enhancing the physical layer security of non-orthogonal multiple access in large-scale networks," IEEE Transactions on Wireless Communications, vol. 16, no. 3, pp. 1656-1672, 2017.

[3] J. Chen, L. Yang, and M.-S. Alouini, "Physical layer security for cooperative NOMA systems," IEEE Transactions on Vehicular Technology, vol. 67, no. 5, pp. 4645-4649, 2018.

[4] B. Zheng, M. Wen, C.-X. Wang, X. Wang, F. Chen, J. Tang, and F. Ji, "Secure NOMA based two-way relay networks using artificial noise and full duplex," IEEE Journal on Selected Areas in Communications, vol. 36, no. 7, pp. 1426-1440, 2018.

[5] A. Arafa, W. Shin, M. Vaezi, and H. V. Poor, "Secure relaying in non-orthogonal multiple access: Trusted and untrusted scenarios," IEEE Transactions on Information Forensics and Security, vol. 15, no. 1, pp. 210-222, 2019.

[6] L. Lv, Z. Ding, Q. Ni, and J. Chen, "Secure MISO-NOMA transmission with artificial noise," IEEE Transactions on Vehicular Technology, vol. 67, no. 7, pp. 6700-6705, 2018.

[7] Y. Feng, S. Yan, Z. Yang, N. Yang, and J. Yuan, "Beamforming design and power allocation for secure transmission with NOMA," IEEE Transactions on Wireless Communications, vol. 18, no. 5, pp. 26392651, 2019.

[8] M. Zeng, N.-P. Nguyen, O. A. Dobre, and H. V. Poor, "Securing downlink massive MIMO-NOMA networks with artificial noise," IEEE Journal of Selected Topics in Signal Processing, vol. 13, no. 3, pp. 685699, 2019.

[9] R. Liu, T. Liu, H. V. Poor, and S. Shamai, "Multiple-input multipleoutput Gaussian broadcast channels with confidential messages," IEEE Transactions on Information Theory, vol. 56, no. 9, pp. 4215-4227, 2010.

[10] M. Vaezi and H. V. Poor, "NOMA: An information-theoretic perspective," in Multiple Access Techniques for 5G Wireless Networks and Beyond, pp. 167-193, Springer, 2019.

[11] S. A. A. Fakoorian and A. L. Swindlehurst, "On the optimality of linear precoding for secrecy in the MIMO broadcast channel," IEEE Journal on Selected Areas in Communications, vol. 31, no. 9, pp. 1701-1713, 2013.

[12] D. Park, "Weighted sum rate maximization of MIMO broadcast and interference channels with confidential messages," IEEE Transactions on Wireless Communications, vol. 15, no. 3, pp. 1742-1753, 2015.

[13] E. Ekrem and S. Ulukus, "Capacity region of Gaussian MIMO broadcast channels with common and confidential messages," IEEE Transactions on Information Theory, vol. 58, no. 9, pp. 5669-5680, 2012.

[14] M. Vaezi, W. Shin, and H. V. Poor, "Optimal beamforming for Gaussian MIMO wiretap channels with two transmit antennas," IEEE Transactions on Wireless Communications, vol. 16, no. 10, pp. 6726-6735, 2017.

[15] M. Vaezi, Y. Qi, and X. Zhang, "A rotation-based precoding for MIMO broadcast channels with integrated services," IEEE Signal Processing Letters, vol. 26, no. 11, pp. 1708-1712, 2019.

[16] G. H. Golub and C. F. Van Loan, Matrix computations, vol. 3. JHU press, 2012.

[17] J. Nocedal and S. Wright, Numerical optimization. Springer Science \& Business Media, 2006.

[18] Q. Li, M. Hong, H.-T. Wai, Y.-F. Liu, W. Ma, and Z.-Q. Luo, "Transmit solutions for MIMO wiretap channels using alternating optimization," IEEE Journal on Selected Areas in Communications, vol. 31, no. 9, pp. 1714-1727, 2013.

[19] S. Asaad, A. Bereyhi, A. M. Rabiei, R. R. Müller, and R. F. Schaefer, "Optimal transmit antenna selection for massive MIMO wiretap channels," IEEE Journal on Selected Areas in Communications, vol. 36, no. 4 , pp. 817-828, 2018.

[20] Y. Wu, R. Schober, D. W. K. Ng, C. Xiao, and G. Caire, "Secure massive MIMO transmission with an active eavesdropper," IEEE Transactions on Information Theory, vol. 62, no. 7, pp. 3880-3900, 2016. 